\title{
Possible Role of CPK-MB in the Early Detection of Alcohol Related Cardiac Damage
}

\author{
Authors
}

\section{Dr Paramveer Singh, Dr Sanjiv S. Kale}

D Y Patil University

Corresponding Author

Dr Paramveer Singh

Flat No. 8, Rohideshwar CHS, Plot No. 6, Gurudwara Marg, Sec 19,

Nerul East, Navi Mumbai - 400706, Maharashtra

Email: pcms747@gmail.com, Mobile: 7506400785

\section{INTRODUCTION}

Alcohol: A potent psychoactive drug, causes acute and chronic changes in all organ systems. Alcohol consumption in large quantities over a long period of time causes cardiac morbidity. Association between excessive consumption and congestive cardiomyopathy has long been known.

${ }^{(1)}$ Patients consuming $>90 \mathrm{~g}$ of alcohol a day for $>5$ years are at risk for the development of asymptomatic Alcoholic Cardiomyopathy. ${ }^{(2)}$ CPK-MB is a sensitive, specific and cost effective test used in diagnosis of acute myocardial infarction. ${ }^{(3)(4)}$ Few studies, in alcoholics show raised levels due to chronic myocardial damage.

\section{NEED FOR THE STUDY}

Cardiac damage in chronic alcoholics has been assessed by 2D Echo and ECG but not much literature is available on the use of CPK-MB. Alcoholic cardiomyopathy, in the early stages, may be completely reversible if recognized and treated early. ${ }^{(5)}$

\section{METHODOLOGY}

Inclusion Criteria:

> Subjects: 30 alcoholic patients attending OPD of a tertiary care center willing to give consent for the study.

$>$ Duration of alcohol use: 10 years or more.

$>$ Amount of alcohol use: $180 \mathrm{ml}$ or more per day.

\section{Exclusion Criteria:}

$>$ Subjects not willing to give consent.

$>$ Alcoholics with pre-existing cardiac morbidity.

$>$ History of polysubstance use.

Materials

$>$ Creatine Phosphokinase MB (CPK-MB): Normal $=0-24 \mathrm{u} /$ liter.

$>$ Electrocardiogram (ECG).

\section{RESULTS}

Out of the 30 subjects enrolled in the study, 28 were males and 2 females. Most of the subjects ${ }^{(15)}$ were in the age group of 36 to 45 years, followed 
by 46 to 55 years ${ }^{(7)}$, followed by 26 to 35 years ${ }^{(6)}$ and finally more than 55 years ${ }^{(2)}$. CPK-MB was elevated in $26(86.6 \%)$ out of the 30 subjects. There were no significant ECG changes in any of the subjects.

\section{DISCUSSION}

High prevalence of elevation of CPK-MB, in 86.6 $\%$ chronic alcoholics looks like a cause and effect relationship. Studies should be done to determine the usefulness of CPK-MB levels as a screening tool to detect early myocardial damage in alcoholics, especially in the absence of clinical and ECG findings. Since ours is a preliminary pilot study, we suggest that, further research should take into consideration other cardiac parameters like 2D Echo to correlate with CPKMB levels. Thus, the affected patients can be detected at an early stage and proper preventive and curative measures can be taken.

\section{REFERENCES}

1. Bridgen W, Robinson J. Alcoholic heart muscle disease. $\mathrm{Br}$ Med J 1964;ii:1283-9

2. Mariann R. Piano. Alcoholic cardiomyopathy: Incidence, Clinical Characteristics, and Pathophysiology. Chest. 2002;121(5): 1638-1650. doi:10.1378/chest.121.5.1638

3. A. F. Smith et al. Creatine kinase MB isoenzyme studies in diagnosis of myocardial infarction. Br Heart J, 1976, 38, 225 32

4. Konttinen and Somer.BMJ, 1973;1:386

5. George E. Burch, Nicholas P. DePasquale. Alcoholic cardiomyopathy The American Journal of Cardiology, Vol 23, Issue 5, 723-31 